\title{
PRIMER REGISTRO DE LA SUBFAMILIA SYCORYCTINAE \\ (HYMENOPTERA: CHALCIDOIDEA: PTEROMALIDAE) \\ Y DEL GÉNERO PHILOTRYPESIS FÖRSTER, 1878, PARA REPÚBLICA DOMINICANA
}

\section{First record of the Sycoryctinae subfamily (Hymenoptera: Chalcidoidea: Pteromalidae) and the genus Philotrypesis Förster, 1878, for the Dominican Republic}

\author{
Candy Ramírez Pérez
}

Instituto de Investigaciones Botánicas y Zoológicas Prof. Rafael M. Moscoso, Universidad Autónoma de Santo Domingo (UASD), Santo Domingo, República Dominicana. ramirez.candy@hotmail.com.

\section{RESUMEN}

Se menciona por primera vez para la República Dominicana y el Caribe la presencia de la subfamilia Sycoryctinae (Chalcidoidea: Pteromalidae) y el género Philotrypesis Förster, 1878. Ejemplares de este grupo fueron encontrados en siconos de Ficus microcarpa L. (Moraceae) en el campus de la sede central de la Universidad Autónoma de Santo Domingo (UASD).

Palabras clave: Ficus, avispas de los higos, Philotrypesis, primer registro, Hispaniola.

\section{ABSTRACT}

The genus Philotrypesis Förster, 1878 of the subfamily Sycoryctinae (Chalcidoidea: Pteromalidae) is reported for the first time in Dominican Republic and the Caribbean. It was found in syconia of Ficus microcarpa L. (Moraceae) at the main campus of the Universidad Autónoma de Santo Domingo (UASD).

Keywords: Ficus, fig wasps, Philotrypesis, first record, Hispaniola.

Dentro de los siconos del género Ficus (Moraceae), comúnmente conocidos como higos, se desarrollan sus polinizadores (Agaonidae), los cuales tienen un mutualismo obligado con este género de plantas. Estos tienen competidores que limitan sus poblaciones y producen efectos que, en algunos casos, pueden ser negativos para las plantas. Se cree que estos inquilinos surgieron poco después de que los polinizadores se irradiaran, hace 49-69 Ma (Segar et al., 2012). Algunos de estos competidores pertenecen a la familia Pteromalidae, himenópteros apócritos de la superfamilia Chalcidoidea, con unas 4100 especies y 640 géneros descritos. Dentro de los Pteromalidae se encuentra la subfamilia Sycoryctinae, la cual pertenecía a la familia de los Agaonidae hasta que un estudio molecular las colocó en esta familia (Rasplus et al., 1998).

Los Sycoryctinae son avispas parasitoides o inquilinos de los siconos de Ficus. Esta subfamilia incluye unos 12 géneros y más de 170 especies descritas, tiene distribución mundial, está registrada como parásita de los subgéneros conocidos de esta planta: Ficus, Sycidium, Sycomorus, Pharmacosycea y Urostigma (van Noort y Rasplus, 2018) y es parásita de casi todos los géneros de la familia Agaonidae. Las especies de Sycoryctinae exhiben ovipositores largos a extralargos y ovipositan desde fuera del sicono (Fig. 1). 
El género Philotrypesis (Fig. 1) es nativo de regiones tropicales y subtropicales del hemisferio oriental (Asia, Irán) y fue introducido en América, según Bouček (1993). La base de datos FigWeb registra unas 50 especies. En América, se cita para Ficus microcarpa (Beardsley, 1998), cuando estos fueron introducidos como árboles ornamentales en muchas ciudades alrededor del mundo. En la década de 1930 fueron introducidos en Hawáii (Beardsley, 1998).

Dentro de la sede central de la Universidad Autónoma de Santo Domingo hay unas cinco especies de Ficus plantados que se encuentran dispersos por todo el campus. Se tomó una de estas especies, Ficus microcarpa, de la cual se encontraron cuatro árboles y se colectó una muestra de cinco siconos de cada uno de ellos, los mismos se llevaron al laboratorio y fueron preservados en alcohol etílico al 70\%. Los siconos fueron abiertos longitudinalmente para ser observados al microscopio y separar e identificar los especímenes, los cuales están depositados en la colección entomológica del Instituto de Investigaciones Botánicas y Zoológicas de la UASD.

Se encontraron avispas de las familias Agaonidae y Pteromalidae. Del género Philotrypesis se encontró un individuo hembra dentro de un sicono. Este hallazgo registra el género Philotrypesis para la República Dominicana y la región del Caribe, contribuyendo así al conocimiento de la diversidad biológica de avispas de los higos. Observaciones y registros de este tipo son muy importantes, ya que permiten conocer las complejidades de las relaciones entre polinizadores y no polinizadores presentes en Ficus microcarpa, además de aumentar el conocimiento existente sobre este mutualismo en la región del Caribe.

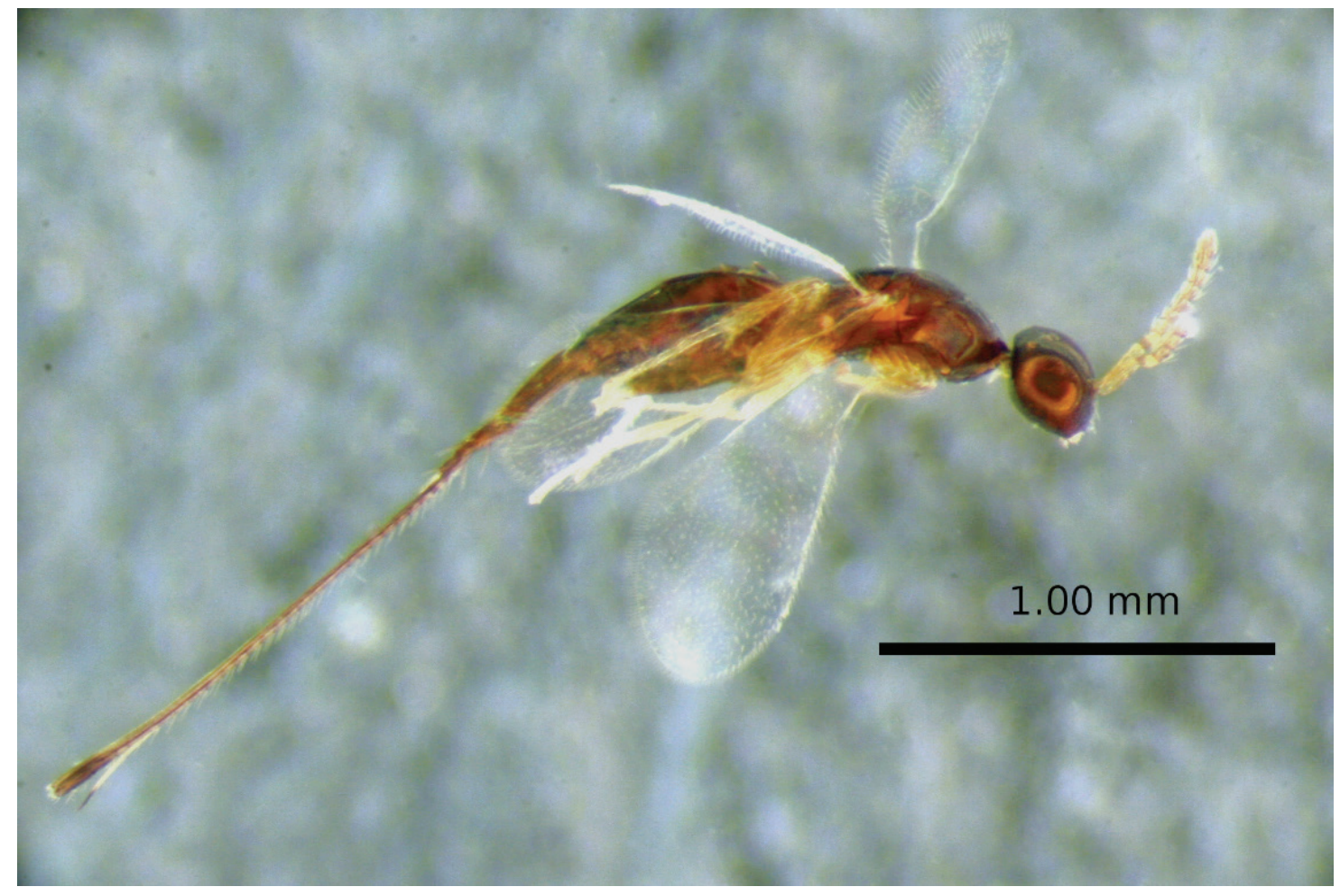

Figura 1. Philotrypesis en Ficus microcarpa. Ejemplar hembra encontrado en siconos de la planta dentro del campus central de la Universidad Autónoma de Santo Domingo (UASD). 


\section{AGRADECIMIENTOS}

América Sánchez ayudó con las colectas y las fotografías, Ruth Bastardo hizo correcciones del manuscrito, mis agradecimientos para ambas colegas del Instituto de Investigaciones Botánicas y Zoológicas “Prof. Rafael M. Moscoso" (UASD) por la colaboración prestada.

\section{LITERATURA CITADA}

Bouček, Z. 1993. The genera of chalcidoid wasps from Ficus fruit in the New World. Journal of Natural History, 27: 173-217.

Beardsley, J. W. 1998. Chalcid wasps (Hymenoptera: Chalcidoidea) associated with fruit of Ficus microcarpa in Hawaii. Proceedings of the Hawaiian Entomological Society, 33: 19-34.

Rasplus, J. Y., C. Kerdelhué, I. Le Clainche y G. Mondor. 1998. Molecular phylogeny of fig wasps Agaonidae are not monophyletic. Académie des sciences. Paris, Sciences de la vie / Life Sciences, 321: 517-527.

Segar, S. T, C. Lopez-Vaamonde, J. Y. Rasplus y J. M. Cook. 2012. The global phylogeny of the subfamily Sycoryctinae (Pteromalidae): Parasites of an obligate mutualism. Molecular Phylogenetics and Evolution, 65: 116-125.

Van Noort, S. y J. Y. Rasplus. 2018. Figweb: higos e higueras avispas del mundo. URL: www. figweb.org, accesado en fecha 10-02-2018.

[Recibido: 30 de mayo, 2018. Aceptado para publicación: 15 de junio, 2018] 